\title{
Energy management for age of information control in solar-powered loT end devices
}

\author{
Abdul Kerim Aydin ${ }^{1} \cdot$ Nail Akar $^{2}$ (1) \\ Accepted: 20 April 2021 / Published online: 12 May 2021 \\ (C) The Author(s), under exclusive licence to Springer Science+Business Media, LLC, part of Springer Nature 2021
}

\begin{abstract}
In this paper, we propose several harvesting-aware energy management policies for solar-powered wireless IoT end devices that asynchronously send status updates for their surrounding environments to a network gateway device. For such devices, we aim at minimizing the average age of information (AoI) metric which has recently been investigated extensively for status update systems. The proposed energy management policies are obtained using discrete-time Markov chain-based modeling of the stochastic intra-day variations of the solar energy harvesting process in conjunction with the average reward Markov decision process formulation. With this approach, energy management policies are constructed by using the time of day and month of year information in addition to the instantaneous values of the age of information and the battery level. The effectiveness of the proposed energy management policies in terms of their capability to reduce the average AoI as well as improving upon the tail of the AoI distribution, is validated with empirical data for a wide range of system parameters.
\end{abstract}

Keywords Internet of things - Solar energy harvesting · Energy management $\cdot$ Age of information $\cdot$ Markov decision processes

\section{Introduction}

Low-Power Wide Area Networks (LPWANs) are suitable for IoT (Internet of Things) infrastructures which are built to serve a massive number of IoT End Devices (ED) each equipped with sensors, (optionally) actuators, and long-range, low-power radios [11]. In LPWANs, the end devices are within a single hop from the so-called Network Gateway (NG) which serves as a bridge for all the IoT end devices to the IP-based Internet. Figure 1 illustrates a single-hop LPWAN serving $\mathbf{N}$ end devices which are in charge of sending status update messages to the NG in the transmit-only configuration which is the focus of this paper. Although bi-directional communication is supported

Nail Akar

akar@ee.bilkent.edu.tr

Abdul Kerim Aydin

akaydin@aselsan.com.tr

ASELSAN, Ankara, Turkey

2 Electrical and Electronics Engineering Department, Bilkent University, Bilkent 06800, Ankara, Turkey in most IoT technologies, we study in this paper the transmit-only scenario where the IoT end devices of interest do not necessarily receive traffic from the gateway. Various LPWAN technologies have been proposed or rolled out in the recent years such as LoRa, SigFox, Ingenu, Weightless, LTE-M, NB-IOT, etc. [11, 26, 37, 39] with different infrastructure choices and service types. Although IoTs may also be viewed as some form of Wireless Sensor Networks (WSN) [3], (i) hardware and software complexity of the IoT end devices needs to be far lower than those used in a typical short-range WSN, (ii) IoT communication infrastructures should support massive connectivity with end devices disseminating information at relatively very low bit rates corresponding to very low duty cycles for each device, (iii) energy consumption requirements on IoT end devices are more stringent.

Considering non-rechargeable battery-powered IoT end devices, frequent human attention would be required to replace the batteries which may neither be desirable nor possible, in many real-life scenarios. Therefore, end devices need to be self-sufficient, i.e., not requiring any human intervention, for a relatively long time [9]. In energy 


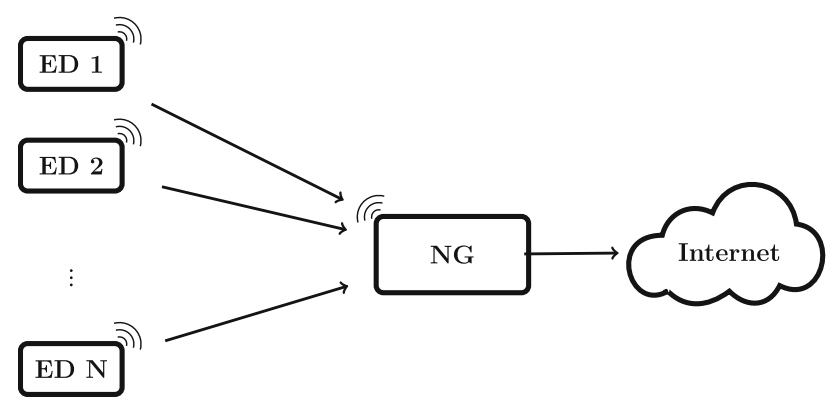

Fig. 1 A single-hop LPWAN serving $\mathbf{N}$ IoT end devices in transmitonly mode. $E D$ end device, $N G$ network gateway

harvesting-based solutions, end devices are equipped to harvest energy from environmental (solar, electromagnetic, wind, etc.) sources and store the harvested energy in the rechargeable batteries $[14,27,51,59,62]$. However, energy harvesting may be quite random in nature and intelligent energy management mechanisms are required for end devices that take into account the statistical properties of the underlying energy harvesting process.

In this paper, we consider an information update system consisting of IoT end devices each equipped with a sensor(s). The state of the device is assumed to change in time which is detected by its sensor and the device occasionally generates information packets that contain sensed data along with a time stamp. The Age of Information (AoI) for a single information source is defined as the time elapsed since the generation of the last successfully received update packet at a remote monitor either held at the NG or the Internet. The AoI concept was first introduced in [29] and later studied in more depth in $[30,31]$ as a metric to quantify the freshness of knowledge about the status of a remote information source in a status update system. There has recently been a surge of interest on AoI-related optimization methods $[1,5,25,49,58]$ in various contexts. [34] provide a relatively recent survey of the AoI concept and its applications. Most existing papers study the continuous-time setting in which the AoI is allowed to take continuous values. However, there has recently been several studies on the discrete-time setting as well, for which the AoI takes discrete values [43, 48, 52]. In the current paper, we follow the discrete-time setting where the AoI is defined as the age in discrete units of time of the most recent status update at the remote monitor for the particular IoT ED of interest.

The focus of this work is on solar-powered end devices in isolation (from other devices) for which the solar energy harvester exhibits temporal variations at different time scales. The goal of the end device is to send status updates as frequently as possible to the NG to keep the information fresh. Figure 2 illustrates an energy harvesting IoT end device sensing status update messages to the gateway.

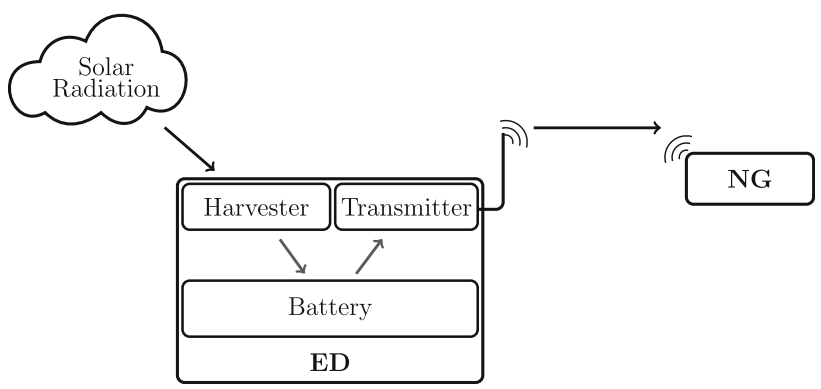

Fig. 2 Solar-powered IoT ED sending status update messages to a NG

The operation of the end device is as follows. The device of interest operates in discrete time by waking up periodically with period $T$ and making a decision to make a status update depending on the current status of the device. Each status update requires a certain energy $E_{s}$ for sensing, processing, and transmission. If the status update decision is positive, the environment is sensed, processed, and an information packet is formed and transmitted towards the NG after which the end device goes back to sleep again. In this paper, we assume that suitable forward error correction mechanisms are in place giving rise to negligible packet error probabilities. Therefore, the effects of the statistical parameters of the communication channel, i.e., bit error rates, signal to noise ratio, etc., as well as MAC-layer characteristics such as collision rates, packet error rates, etc., are deliberately left outside the scope of the current paper. In case the status update decision is negative, the ED goes back to sleep immediately while consuming an energy $E_{f}<<E_{s}$. Since the slot time $T$ would typically be much longer in low duty cycle IoT networks compared to the transmission time of the information packet and network delay, we will assume throughout the paper that the information packets carrying status update messages are delivered immediately upon the sensing event. In Fig. 3, the AoI process $\Delta_{k}$ is illustrated as a function of the integer time index $k$ that keeps track of time in units of time slots of length $T$ in an example scenario. In this figure, $A_{i}, i \geq 0$ represents the status update instance for the $i$ th status

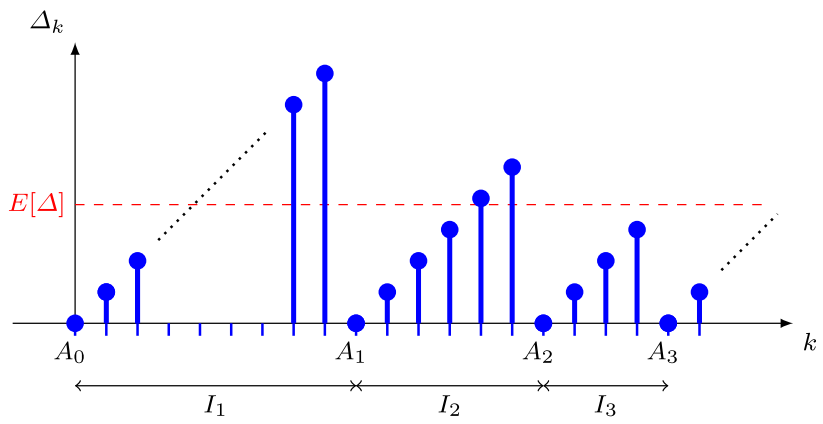

Fig. 3 A sample path for the AoI process $\Delta_{k}, k \geq 0$. Status updates occur at instances $0,9,15$, and 19 in this example 
update, and $I_{i}$ denotes the inter-update time between the status updates $i-1$ and $i$. Every time a status update decision is made, the AoI process $\Delta_{k}$ is brought down to zero. On the other hand, the AoI process $\Delta_{k}$ is incremented at each time slot until the next status update. The Average AoI (AAoI), also denoted by $E[\Delta]$, is defined as the following time average due to the ergodicity of the AoI process $\Delta_{k}$ :

$$
E[\Delta]=\lim _{K \rightarrow \infty} \frac{\sum_{k=0}^{K-1} \Delta_{k}}{K} .
$$

In the current paper, we propose to develop various status update policies for an edge device in isolation so as to minimize the single metric $E[\Delta]$ as a function of the current state of the edge device given in terms of some or all of the following: (i) current battery energy, (ii) current AoI value, (iii) time of day, (iv) month of year.

Various energy management algorithms have been developed in the literature for different types of energy harvesting sources and different metrics. In particular, adaptive duty cycling methods that adaptively adjust the inter-sensing times have been developed to maximize system performance while avoiding power failures [8, 20, 24, 28, 40, 46, 57, 63]. Energy harvesting sources usually have a stochastic nature which can well be represented with Markov Chains (MC) [40]. In such cases, Markov Decision Processes (MDP) have been proposed towards the optimal energy management of energy harvesting SNs when the underlying stochastic model is MCbased [4].

In this paper, based on real datasets for solar harvesting, we model the intra-day variations of the energy harvesting process of a solar-powered IoT ED by a Discrete-Time MC (DTMC). Additionally, we propose to use the average reward MDP framework and the Policy Iteration (PI)-based DP (Dynamic Programming) method to obtain status update policies so as to minimize $E[\Delta]$. The main contributions of this paper are the following:

- Most existing studies employ generic energy harvesting sources with no emphasis on the specific attributes of the harvesting source. In this paper, we focus on solarpowered energy harvesting sources and propose a novel method for Markov chain modeling of the intra-day variations of a solar source using empirical data. In this method, a single day is divided into a certain number of time intervals so as to keep track of the solar radiation patterns at different times of the day. For example, when two time intervals within a day are to be used, then one can differentiate between daytime and nighttime radiation patterns. When the number of time intervals is to be increased, the proposed Markov chain is able to model more accurately the intra-day variations of the solar source. With the proposed MDP formulation for AAoI minimization, the time of day information is naturally used by the energy manager through the state of the Markov chain corresponding to the particular time interval of a given day. Using the time of day information in the energy manager significantly enhances the AoI performance of the device as shown through numerical examples.

- For solar sources, seasonal (or monthly) variations of the solar source are also critical for the energy manager. The energy management policy to be employed during Winter days of low solar radiation should be different than a policy to be used in Summer days with high solar radiation. In this paper, we propose a new method to effectively take into account such seasonal variations, with acceptable complexity and storage requirements, for the proposed energy manager. In this proposed method, optimum policies are obtained for each month of the year with the Policy Iteration approach and the energy manager employs policy switching at different months of the year. Using the month of year information in the energy manager is shown to have a key effect on the AoI performance of the end device.

- Two different 20-year long datasets belonging to two different locations are studied extensively for developing an energy management policy for each of two locations and consequently, we validate the effectiveness of this approach for both locations. We show that using the time of day and month of year information (in addition to current battery level and instantaneous AoI) in the transmission policy is remarkably effective in reducing AAoI and also the AoI violation probabilities.

The paper is organized as follows. In Sect. 2, related work on energy management of energy harvesting end devices is presented. MDPs are briefly described in Sect. 3 along with the PI algorithm used in this paper. In Sect. 4, the MDP formulation for the purpose of AAoI minimization is presented whereas the DTMC model of the solar harvesting process and various status update policies obtained from the MDP formulation are presented in Sect. 5. In Sect. 6, numerical examples are given for two different solar datasets that validate the effectiveness of the proposed approach. Finally, we conclude.

\section{Related work}

Energy management of energy harvesting wireless sensor nodes has been a topic of research interest in recent years; see the references $[2,33,56]$ for surveys in this area. From a general perspective, these energy management algorithms may be categorized into two groups: (i) offline problem and 
(ii) online problem. The offline problem assumes the availability of knowledge on energy arrivals prior to the system execution; then, the energy management task turns into a planning problem. Reference [60] focuses on a system where energy and data packet sizes are known and the aim is to minimize the transmission completion time. [55] present optimal solutions for throughput maximization for the offline case with consideration of storage losses due to the battery imperfections. A group size based approach to adaptively manage the duty cycle of sensor nodes is proposed to prolong network lifetime in [12].

In the online scenario, data and energy arrivals are not known prior to operation but they both arrive according to a statistical model. A recent work in [17] investigates the stochastic model of a general computing system where the energy arrivals and job arrivals are both random. In [38], a threshold-based policy is proposed to maximize an average reward function in the long-term in a setting where the harvesting policy is modeled as a two-state Markov chain. A power consumption planning scheme is constructed by [54] with the assumption of a deterministic energy arrival process. The study in [36] also considers the number of packets waiting in the queue as another dimension to the Markov chain. Some prediction-based energy managers [10] use predictions of the future amount of harvested energy in a finite time horizon for deciding on the energy consumption of the node. There are also some studies which focus on optimizing the energy consumption of sensor nodes with the objective of energy-neutral operation by modeling the harvesting process as a MDP [18, 47]. While the main focus in these energy-oriented schemes is to prolong the network lifetime by controlling the node duty cycles, QoS requirements are not taken into consideration [22].

For the online energy management schemes, adaptive duty cycling is a commonly used strategy for optimizing the energy consumption of a sensor node according to the state of the energy and data sources by making the nodes sleep or wake at proper times to use the energy of the node efficiently. Reference [28] proposes a mixed approach that assumes a periodic discrete model for the harvested energy to decide on the duty cycles based on this model and adjusts the duty cycles online with deviations of the real harvested energy from the estimate. In [18], optimal energy management algorithms are proposed to maximize the data rate of the node in the long run for several energy storage and harvesting models. [58] focus on AoI minimization in a status update scheme with finite and infinite battery cases and they offer a threshold based approach for minimizing the AoI. [6] propose a multi-threshold structure for energy management for which the sensor sends an update only if the age of information grows above a certain threshold that depends on the available energy. The problem of finding an age-optimal threshold policy is studied in [7] with the transmission threshold being a function of the energy state and the estimated current age. [16] study an optimal online status updating policy to minimize the AAoI at the destination for a scenario where the communications channel is noisy and there is a non-zero probability of failures for status updates. Our work is different than the age-optimal schemes studied in the literature is that we also use the time of day and month of year information due to the intra-day and inter-monthly variations that are known to exist for the solar harvesting process.

There have also been research studies specific to solarpowered wireless sensor networks. [32] propose a solar energy harvesting based MAC protocol which is adaptive to the changing weather conditions for smart agriculture applications. A machine learning based adaptive duty cycle algorithm for energy harvesting wireless sensor networks is proposed by [45] using solar forecasting. The trade-off between energy and performance is addressed by [21] via an MDP formulation of a simple fully solar-powered case study with finite states representing levels of battery charge and solar intensity.

Stochastic modeling of the energy harvesting process has been another area of research. In [44], a day is divided into time slots with the harvesting power and consumption in each slot being known and constant. [15] extend this approach with the objective of maximizing the amount of data transmitted within a specified finite time horizon. In [53], the node battery level is represented with a Markov fluid queue relying on a Continuous Time Markov Chain (CTMC) that models the harvesting process. [28] model the harvesting and consumption as two independent bounded random processes. The reference [61] assumes a two-state (active and passive) continuous-time Markovian model with independent exponential random variables to represent the duration of stay in each state.

\section{Discrete-time Markov decision processes}

The following brief discussion on discrete-time Markov Decision Processes (MDP) is based on [50] and [19]. Consider a controlled DTMC $X_{k}^{\pi}, k=1,2, \ldots$, with finite state-space $\mathcal{S}=\{1,2, \ldots, N\}$ for each policy $\pi \in \Pi$ which amounts to a mapping from the state-space $\mathcal{S}$ to the action space $\mathcal{A}$ for which a deterministic action from the set $\mathcal{A}_{i} \subset$ $\mathcal{A}=\{1,2, \ldots, M\}$ is to be taken when the underlying Markov chain is at state $i \in \mathcal{S}$ and this policy does not change with respect to time. Observe that the cardinality of the entire policy set $|\Pi|=M^{N}$ for the case $\mathcal{A}_{i}=\mathcal{A}$ for all $i$, which impedes operations with exhaustive search-based methods to find optimal policies. We use the notation 
$p(i, a, j)$ to denote the one-step transition probability of transitioning from state $i$ to state $j$ under action $a \in \mathcal{A}_{i}$ for $i, j \in \mathcal{S}$. When policy $\pi$ is followed, the transition probability of going from $i$ to $j$ is denoted by $p(i, \pi(i), j)$ with an immediate reward $r(i, \pi(i), j)$ collected as an outcome of this transition. Let

$$
r^{\pi}(i)=\sum_{j} r(i, \pi(i), j) p(i, \pi(i), j)
$$

denote the expected immediate reward collected when policy $\pi$ is applied at state $i$.

Stemming from the definition of AAoI, the focus of this paper will be average-reward infinite-horizon MDPs for which one is charged with the task of finding an optimal policy $\pi^{*} \in \Pi$ to maximize the expected reward per unit time over an infinite horizon. Let us assume the regularity of the DTMC $X_{k}^{\pi}, k=1,2, \ldots$ when the policy $\pi$ is applied so that the unique limiting probability $x^{\pi}(i)=$ $\lim _{k \rightarrow \infty} \operatorname{Pr}\left\{X_{k}^{\pi}=i\right\}, i=1, \ldots, N$ exists [19]. In this case, the optimum policy $\pi^{*}$ is obtained as the solution of the following optimization problem:

$$
\pi^{*}=\operatorname{argmax}_{\pi \in \Pi} \rho^{\pi}=\operatorname{argmax}_{\pi \in \Pi} \sum_{i} x^{\pi}(i) r^{\pi}(i),
$$

where $\rho^{\pi}$ denotes the average reward collected by the DTMC $X_{k}^{\pi}$.

The Policy Iteration (PI) algorithm of [23] for solving the optimization problem (3) is given below.

Step 1: Set $k=1$ and start with an arbitrary policy $\pi_{1}$.

Step 2: (Policy Evaluation) Evaluate the policy $\pi_{k}$ to obtain $\rho^{\pi_{k}}$ and the following value functions $h^{k}(i), i=1, \ldots, N$ :

$$
h^{k}(i)=r^{\pi_{k}}(i)-\rho^{\pi_{k}}+\sum_{i=1}^{N} p\left(i, \pi_{k}(i), j\right) h^{k}(j) \text {. }
$$

Step 3: (Policy Improvement) Choose a new policy $\pi_{k+1}$ such that

$$
\pi_{k+1}(i)=\operatorname{argmax}_{a \in \mathcal{A}_{i}} \sum_{j} p(i, a, j)\left(r(i, a, j)+h^{k}(j)\right) .
$$

Step 4: If $\pi_{k+1}=\pi_{k}$, then set $\pi^{*}=\pi_{k+1}$ and stop. Otherwise, set $k=k+1$ and go to Step 2 .

Implementation details of the PI algorithm are given in [19] and [23]. We propose to use this PI algorithm to solve average reward MDPs in this paper.

\section{MDP model for the energy manager}

For the MDP model for the energy manager, we take the basic energy unit as $e \mathrm{mWh}$ and all the other energy quantities of interest will be assumed to be integer multiples of this basic unit. Subsequently, we assume a battery with a finite capacity $B$, i.e., it can store at most $B e \mathrm{mWh}$. The IoT end device wakes up at the beginning of each time slot and decides whether or not to fulfill the combined status update task of sensing, processing, and transmission. We assume that this task gives rise to the consumption of $E_{s} \mathrm{mWh}$ of energy when such action is taken and the actual time duration required to complete this task will be negligibly small compared to the time slot duration which is defined as the sleep duration between actions. We also define $L_{s}=E_{s} / e$. We also assume $E_{f} \mathrm{mWh}$ of fixed energy consumption which is required for waking up and processing required to take an action. Similarly, we define the integer $L_{f}=E_{f} / e$ that is to be used in the MDP formulation. Obviously, the parameters $L_{s}$ and $L_{f}$ are assumed to take integer values stemming from the discrete-time setting of our proposed approach. Note that the basic energy unit $e$ can always be chosen arbitrarily small so that such discretization does not give rise to an unacceptable inaccuracy.

The harvesting process is assumed to be governed by a DTMC with $N$ states with probability transition matrix $Q$ with its $(i, j)$ th entry denoted by $Q(i, j)$. In the next section, we will describe a method of obtaining such DTMCs. At state $n$ of this DTMC, $n=1,2, \ldots, N$ of this DTMC, we assume $\ell$ units of energy will be harvested during a time slot with probability $p(n, \ell)$ for $\ell=0,1, \ldots, L$, where $L$ denotes the maximum amount of harvestable energy during a time slot, provided the battery limit has not been reached.

Recall that AoI is defined as the elapsed time slots since the last status update of the IoT device and our goal is to minimize the AAoI. To model this system, we propose the following three-dimensional discrete-time MDP composed of the following components: the discretized value of the remaining energy in the battery $X_{B}(k) \in \mathcal{B}=\{0,1, \ldots, B\}$, the value of the age of information $X_{I}(k) \in \mathcal{I}=\{0,1, \ldots, I\}$ in terms of time slots, and the harvester DTMC $X_{N}(k) \in \mathcal{N}=\{1,2, \ldots, N\}$, at the beginning of time slot $k$, where a finite value $I$ is enforced to keep the overall MDP finite-dimensional; see [35] for data-driven stochastic models for energy harvesters. Subsequently, we form our combined state space as $\mathcal{S}=\mathcal{B} \times \mathcal{I} \times \mathcal{N}$. The action space for the states can be written as $\mathcal{A}=\{1,0\}$, where 1 and 0 represent whether or not a status update is made, respectively, in the corresponding state. Note that when $X_{B}(k)<L_{f}+L_{s}$, then the action $A(k)$ can not be 1 . While the transition probability of 
the battery state depends on the harvester state, state transition probabilities for the AoI state depend only on the action taken in that state. Subsequently, the stationary transition probability from state $\left(X_{B}(k), X_{I}(k), X_{N}(k)\right)=$ $(b, i, n)$ to state $\left(X_{B}(k+1), X_{I}(k+1), X_{N}(k+1)\right)=$ $\left(b^{\prime}, i^{\prime}, n^{\prime}\right)$ at the $k$ th time slot, denoted by $p_{b, i, n}^{b^{\prime}, i^{\prime}, n^{\prime}}$ can be written as the following product:

$$
\begin{aligned}
p_{b, i, n}^{b^{\prime}, i^{\prime}, n^{\prime}}= & \operatorname{Pr}\left\{X_{I}(k+1)=i^{\prime} \mid X_{I}(k)=i\right\} \\
& \cdot \operatorname{Pr}\left\{X_{B}(k+1)=b^{\prime} \mid\left(X_{B}(k), X_{N}(k)\right)=(b, n)\right\} \\
& \cdot \operatorname{Pr}\left\{X_{N}(k+1)=n^{\prime} \mid X_{N}(k)=n\right\} .
\end{aligned}
$$

The transition probability to $X_{I}(k+1)=i^{\prime}$ from $X_{I}(k)=i$ is dependent on the action value $A(k) \in \mathcal{A}$. When $A(k)=1$, this relation can be written as:

$\operatorname{Pr}\left\{X_{I}(k+1)=i^{\prime} \mid X_{I}(k)=i\right\}=\left\{\begin{array}{lc}1, & \text { if } i^{\prime}=0, \\ 0, & \text { otherwise }\end{array}\right.$

When $A(k)=0$ and $i<I$,

$\operatorname{Pr}\left\{X_{I}(k+1)=i^{\prime} \mid X_{I}(k)=i\right\}=\left\{\begin{array}{cc}1, & \text { if } i^{\prime}=i+1, \\ 0, & \text { otherwise. }\end{array}\right.$

If $i=I$ when $A(k)=0$, then $X_{I}(k+1)$ is forced to stay at $I$ with probability 1 .

The battery level evolves according to the following equation for $k \geq 0$ :

$X_{B}(k+1)=\min \left(B, \max \left(0, X_{B}(k)-L_{f}\right)-L_{s} A(k)+X_{N}(k)\right)$,

and $A(k)$ should be zero when $X_{B}(x)<L_{f}+L_{s}$. Let $p_{b, n}^{b^{\prime}}$ denote the probability $\operatorname{Pr}\left\{X_{B}(k+1)=b^{\prime} \mid\left(X_{B}(k), X_{N}(k)\right)\right.$ $=(b, n)\}$. For the control action $A(k) \in\{0,1\}$, we have

$p_{b, n}^{b^{\prime}}=\left\{\begin{array}{cl}p\left(n, b^{\prime}-b+L_{f}+L_{s} A(k)\right), & \text { if } b^{\prime}<B, \\ \sum_{\ell=B-b+L_{f}+A(k) L_{s}}^{L} p(n, \ell), & \text { if } b^{\prime}=B .\end{array}\right.$

Stemming from the DTMC model of the energy harvesting process,

$\operatorname{Pr}\left\{X_{N}(k+1)=n^{\prime} \mid X_{N}(k)=n\right\}=Q\left(n, n^{\prime}\right)$,

irrespective of the action. In the next section, a specific DTMC model for the energy harvesting process will be proposed and described in detail. On the other hand, rewards of the MDP model are to be assigned towards the objective of minimizing AAoI in the long run. For this purpose, we propose to collect a negative reward $-i$ when the MDP resides in a state with $X_{I}(k)=i$. With this reward assignment, the discrete-time MDP formulation is complete with descriptions of the state space, action space, transition probabilities and rewards. In the next section, we describe various data-driven DTMC models to capture the intra-day variations in the amount of harvested energy using solar data and the subsequently obtained energy management policies.

\section{Energy management policies}

In order to obtain an energy management policy using the discrete-time MDP framework described in the previous section, we first need a data-driven DTMC model for the solar energy harvesting process $X_{N}(k)$. For this purpose, let $T$ denote the slot length in minutes and let $Z(d, \sigma)$ be the actual amount of energy harvested at day $d$ for $d=$ $1,2, \ldots, D$ and in slot $\sigma$ for $\sigma=1,2, \ldots, \Sigma$ from historical solar data where $T \Sigma=1440$ amounts to the number of minutes in a single day. Let $z(\sigma)$ denote the average harvested energy (over $D$ days) during slot $\sigma$ in units of the basic energy unit $e$ :

$z(\sigma)=\frac{1}{D e} \sum_{d=1}^{D} Z(d, \sigma)$

with $z_{w}(\sigma)\left(z_{f}(\sigma)\right)$ being the whole (fractional) part of $z(\sigma)$. Since $z(\sigma)$ is real-valued, we define the following

$p(\sigma, \ell)=\left\{\begin{array}{cc}z_{f}(\sigma), & \text { if } \ell=z_{w}(\sigma)+1 \\ 1-z_{f}(\sigma), & \text { if } \ell=z_{w}(\sigma) \\ 0, & \text { otherwise }\end{array}\right.$

as the probability of harvesting $\ell$ discrete units of energy during slot $\sigma$. Next, we partition the $\Sigma$ slots in one single day into $N$ non-overlapping partitions (or intervals) each holding $m$ consecutive time slots such that $\Sigma=m N$. Note that the partitioning parameter $N$ needs to be chosen so that the parameter $m$ turns out to be an integer. Mathematically, the $n$th interval of slots denoted by $I_{n}, n=1,2, \ldots, N$ stands for the following subset of time slots:

$\{m(n-1)+1, m(n-1)+2, \ldots, m n\}$,

where the operations above are modulo $\Sigma$. We then define the following expression

$p(n, \ell)=\sum_{\sigma \in I_{n}} \frac{p(\sigma, \ell)}{m}$

for the empirical probability of harvesting $\ell$ discrete units of energy in the $n$th time interval $I_{n}$ of a given day. Although each of the intervals consists of exactly $m$ time slots, we further approximate the interval duration to be geometrically distributed with mean $m$. Subsequently, we have a DTMC model for the energy harvesting process 
$X_{N}(k) \in \mathcal{N}=\{1,2, \ldots, N\}$ with a probability transition matrix $Q$ of the form

$$
\begin{aligned}
& Q(n, n+1)=Q(N, 1)=\frac{1}{m}, n=1,2, \ldots, N-1, \\
& Q(n, n)=1-\frac{1}{m}, n=1,2, \ldots, N,
\end{aligned}
$$

where at each state $n$, we have a probability $p(n, \ell)$ (given in Eqn.14) of harvesting $\ell$ units of energy. Now that the DTMC model is established, the MDP model of the previous section can be solved to obtain optimal policies to minimize the AAoI in case the DTMC model is exact. However, since the DTMC model is not exact, the produced policies will definitely not be optimal but the effectiveness of these policies will be studied extensively in the next section.

We are now ready to propose the energy management policies we propose to study in this paper. The policy named as the Solar Energy Manager SEM- $N$ uses the DTMC model with $N \geq 1$ states described above for the energy harvesting model to capture the intra-day variations in the solar-radiated energy and feeds it to the PI algorithm for the 3-dimensional MDP of Sect. 4 to generate energy management policies as a function of the instantaneous energy level, instantaneous AoI, and also the current state of the DTMC. Whenever the developed policies are to be evaluated using historical solar data, the actual time of day information, i.e., time interval information, is to be used instead of the state of the corresponding DTMC. Obviously, SEM-1 does not capture the intra-day variations and the time of day information is not used at all in SEM-1. By increasing the number of partitions $N$, the intra-day variations are captured more effectively but at the expense of additional computational complexity for solving the MDP.

The distribution of the harvested energy not only depends on the time of day but also on the month of the year. In order to use the month of year information without having to increase the complexity of the MDP, we propose the following. Let $Z^{v}(d, \sigma)$ be the historical harvested energy information for slot $\sigma$ on day $d$ which is now restricted to month $v=1,2, \ldots, 12$. Stemming from the historical data $Z^{v}(d, \sigma)$, we produce a separate SEM- $N$ policy for each month $v=1,2, \ldots, 12$, using the usual 3-dimensional MDP. When the decisions are to be taken in the on-line scenario, the SEM- $N$ policy is to be used corresponding to the instantaneous month of the year, using the so-called policy switching method. To clarify, one would have a separate policy (obtained with PI) stored for each month and we would resort to a new policy when a month just gets to start. We call the policy switching-based method as MSEM- $N$ (Monthly-switched SEM- $N$ ). Clearly, MSEM- $N$ has 12 times the computational complexity and storage requirements in comparison to SEM- $N$. The number of sunny days in a year in ATL (SEA) is much more
Fig. 4 MSEM-2 transmission policy (SEA dataset when $L_{s}=$ 20 and $B=500$ ) as a function of the instantaneous battery energy level and instantaneous AoI for the following four cases a 7 pm-7 am in January b 7 pm-7 am in July c 7 am-7 pm in January $\mathbf{d} 7$ am-7 pm in July. Light green (dark brown) points indicate a transmission (not transmission) decision (Color figure online) (a)

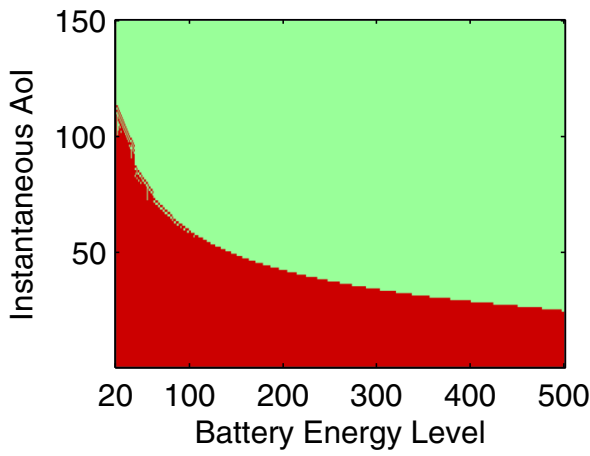

(c)

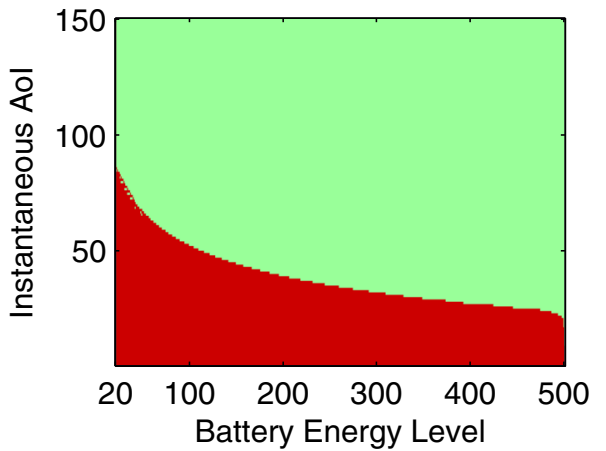

(b)

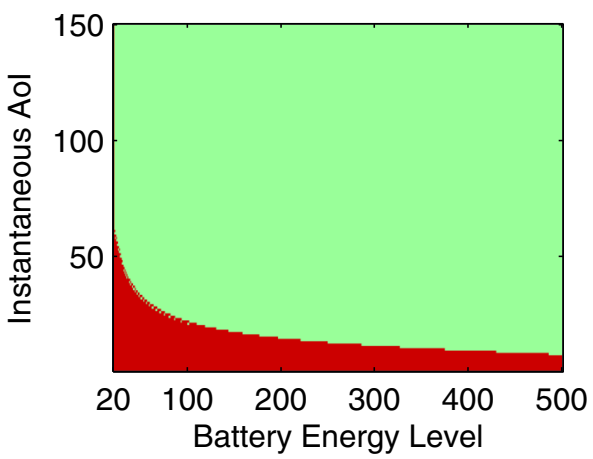

(d)

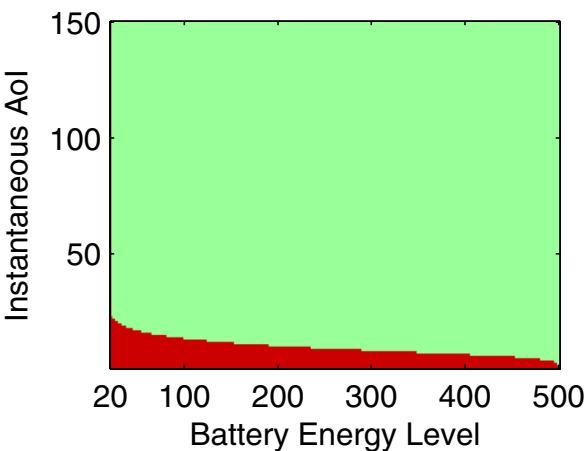


(a) $B=100, L_{s}=1$

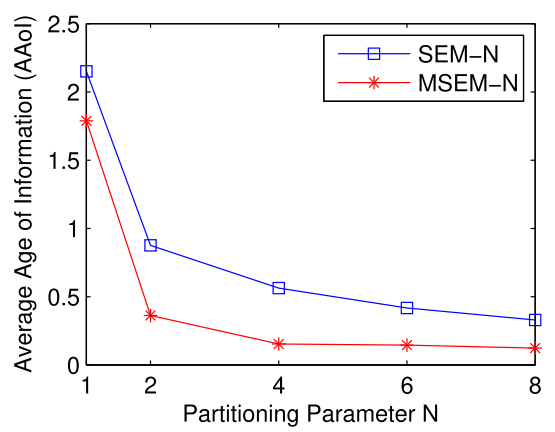

(d) $B=500, L_{s}=1$

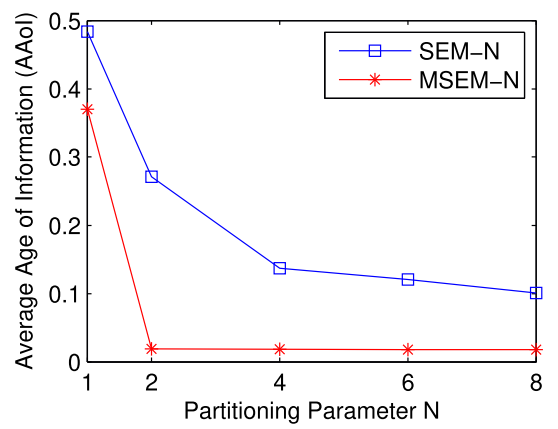

(b) $B=100, L_{s}=5$

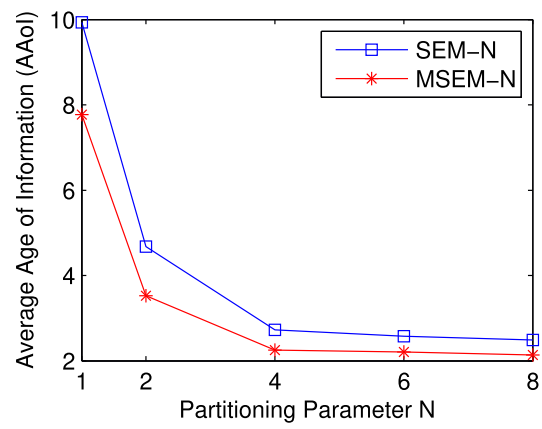

(e) $B=500, L_{s}=5$

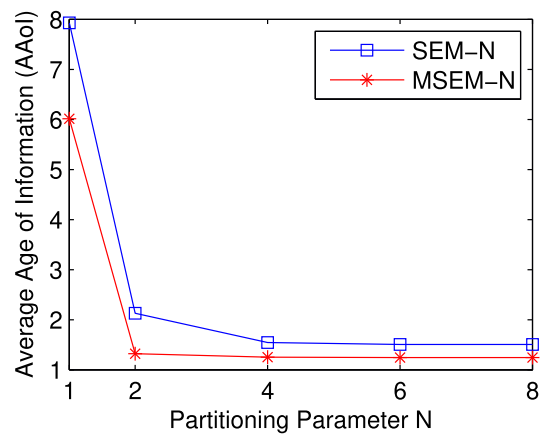

(c) $B=100, L_{s}=20$

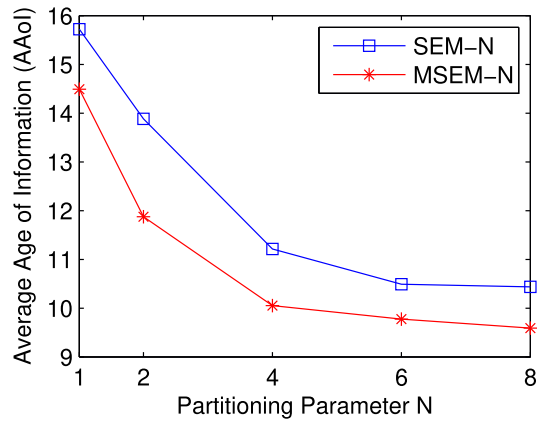

(f) $B=500, L_{s}=20$

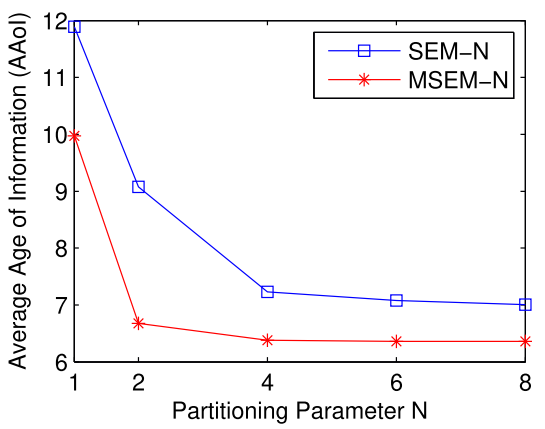

Fig. 5 Average Age of Information (AAoI) obtained with the policies SEM- $N$ and MSEM- $N$ as a function of the partitioning parameter $N$ for the dataset ATL for various values of $B$ and $L_{s}$

(significantly less) than the US average. Therefore, these two datasets exhibit moderately different statistical characteristics. In the next section, we study the performance of the proposed solar energy managers SEM- $N$ and MSEM$N$ for various values of the partitioning parameter $N$ using these two sufficiently long datasets of radiated energy from two different locations on earth.

\section{Numerical examples}

\subsection{Setting}

In the numerical examples, we fix the time slot duration $T=10 \mathrm{~min}$, basic energy unit $e=1 \mathrm{mWh}$, maximum AoI parameter $I=150$, and wake-up energy parameter $L_{f}=0$, whereas we study the effect of other system parameters, namely the sensing and transmission energy parameter $L_{s}$, battery capacity $B$, and the partitioning parameter $N$, on the system performance in terms of AAoI. In order to assess the effectiveness of our approach, we use twenty-year long datasets obtained from the National Solar Radiation Database (NSRDB) [41] which provides solar radiation data at hourly resolution. To interpolate between samples within a single hour, we assume that the solar radiance density is constant throughout that hour. All test cases are repeated with solar radiation data pertaining to two distinct locations in the US, namely the Atlanta Hartsfield International Airport (referred to as dataset ATL) and the Seattle Tacoma International Airport (referred to as dataset SEA). Note that both locations are in middle latitudes for both of which significant intra-day variations exist in solar radiation data. We use the following expression in [42] to obtain $Z(d, \sigma)$ from the solar radiation data for both of the locations:

$Z(d, \sigma)=A_{p} K_{p} S(d, \sigma)\left(1-0.005\left(T_{e}-25^{\circ} \mathrm{C}\right)\right)$,

where $A_{p}$ is the panel area, $K_{p}$ is the panel efficiency factor, $T_{e}$ is the environment temperature in ${ }^{\circ} \mathrm{C}$, and $S(d, \sigma)$ is the solar radiance level in time slot $\sigma$ of day $d$ in appropriate units. In our numerical experiments, we assume a solar panel with area $A_{p}=30 \mathrm{~cm}^{2}, K_{p}=20 \%$, and $T_{e}=25^{\circ} \mathrm{C}$.

While partitioning a day into $N$ intervals, we aim to maximize the difference in the daylight radiation levels between different partitions. Therefore, we split the day such that the peak hour of the day in terms of radiation level, namely 13:00, resides in the center of one of the partitions, say $I_{1}$. For example, in the case of $N=2$ and $N=4, I_{1}$ will consist of all the 10-min time slots between 7 am and 7 pm, and between 10 am and 4 pm, respectively. 
(a) $B=100, L_{s}=1$

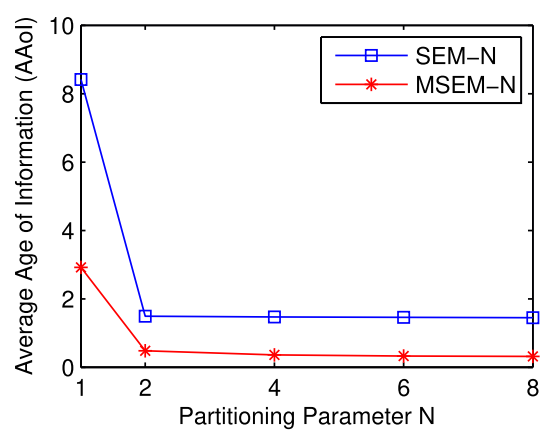

(d) $B=500, L_{s}=1$

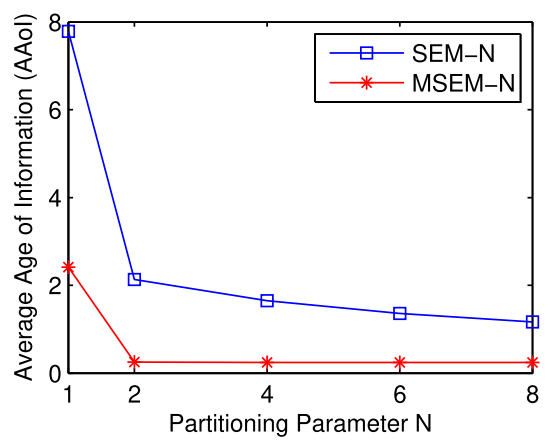

(b) $B=100, L_{s}=5$

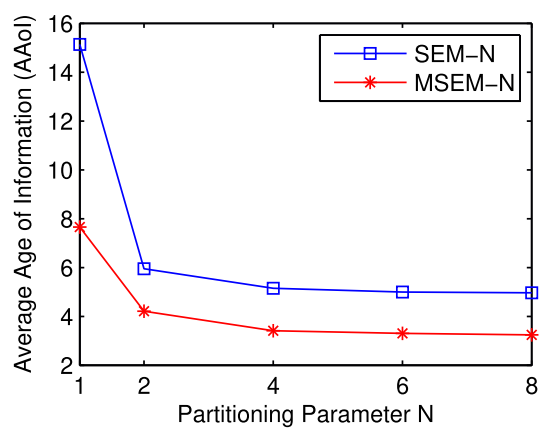

(e) $B=500, L_{s}=5$

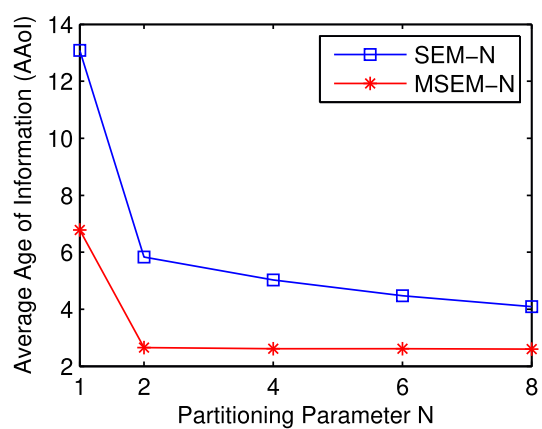

(c) $B=100, L_{s}=20$

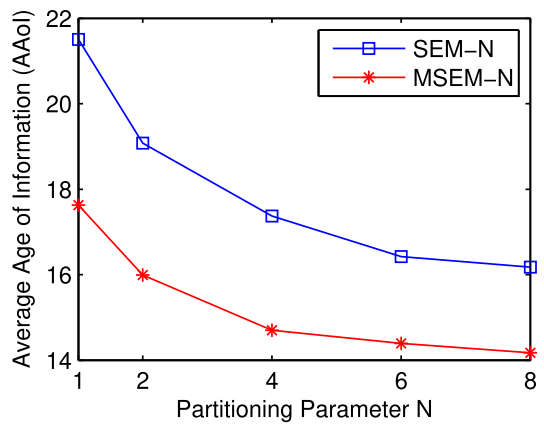

(f) $B=500, L_{s}=20$

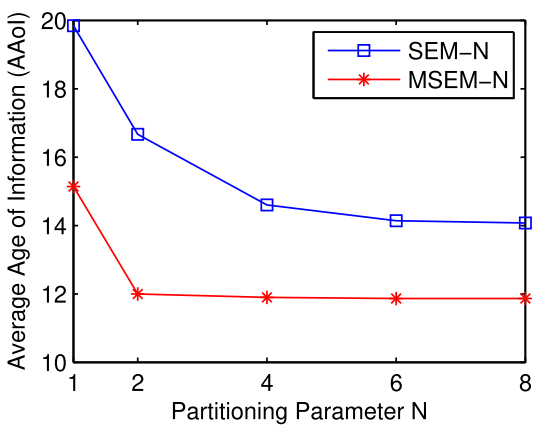

Fig. 6 Average Age of Information (AAoI) obtained with the policies SEM- $N$ and MSEM- $N$ as a function of the partitioning parameter $N$ for the dataset SEA for various values of $B$ and $L_{s}$

Table 1 Percentage AAoI reduction with MSEM-4 compared to SEM-4

\begin{tabular}{llllll}
\hline Dataset & $B$ & \multicolumn{4}{l}{ Improvement in AAoI $(\%)$} \\
\cline { 3 - 6 } & & $L_{s}=1$ & $L_{s}=5$ & $L_{s}=10$ & $L_{s}=20$ \\
\hline ATL & 100 & 72.6131 & 17.5803 & 13.0993 & 10.3484 \\
& 500 & 86.5479 & 19.3695 & 15.5620 & 11.7682 \\
SEA & 100 & 75.7445 & 33.7392 & 24.6557 & 15.3989 \\
& 500 & 85.1143 & 48.0696 & 27.2739 & 18.5223 \\
\hline
\end{tabular}

Moreover, in the actual simulations, we do not impose a bound on the peak AoI value whereas the policies are to be produced with $I=150$ for numerical tractability.

\subsection{Validation of the proposed policies}

In the first example, the structure of a representative transmission policy MSEM-2 obtained with the PI algorithm is depicted in Fig. 4 for two months of the year, i.e., January and July, for the SEA dataset when $L_{s}=20$ and $B=500$. It is clear that the obtained policies for different months and for different partitions of a single day exhibit substantial discrepancies. We also note that for a given partition of a day, the optimum policy is a threshold policy, i.e., at a given value of the battery level, there exists a battery level-dependent AoI threshold such that when the instantaneous AoI exceeds that threshold, the optimum decision is to make a status update and not to make an update otherwise. Moreover, this battery level-dependent threshold decreases with increased battery level. A noteworthy implication of this observation for memory-constrained devices is that the memory required to store the optimal transmission policies can be reduced by storing the thresholds only or the curves obtained in Fig. 4 can further be approximated by piece-wise constant curves with much fewer jumps with further reductions in storage requirements.

In the next example, the transmission policies SEM- $N$ and MSEM- $N$ are obtained using the PI algorithm as a function of the partitioning parameter $N \in\{1,2,4,6,8\}$ for various values of $B$ and $L_{s}$ for both datasets ATL and SEA, and the corresponding AAoI figures are depicted in Figs. 5 and 6, respectively. Our main observation is that for a fixed choice of $N$, the policy MSEM- $N$ substantially outperforms SEM- $N$ which underlines the effectiveness of monthly policy switching. This is not surprising since with MSEM- $N$, one switches to more conservative policies in Winters when solar radiation figures are considerably less, 
(a) SEM-4

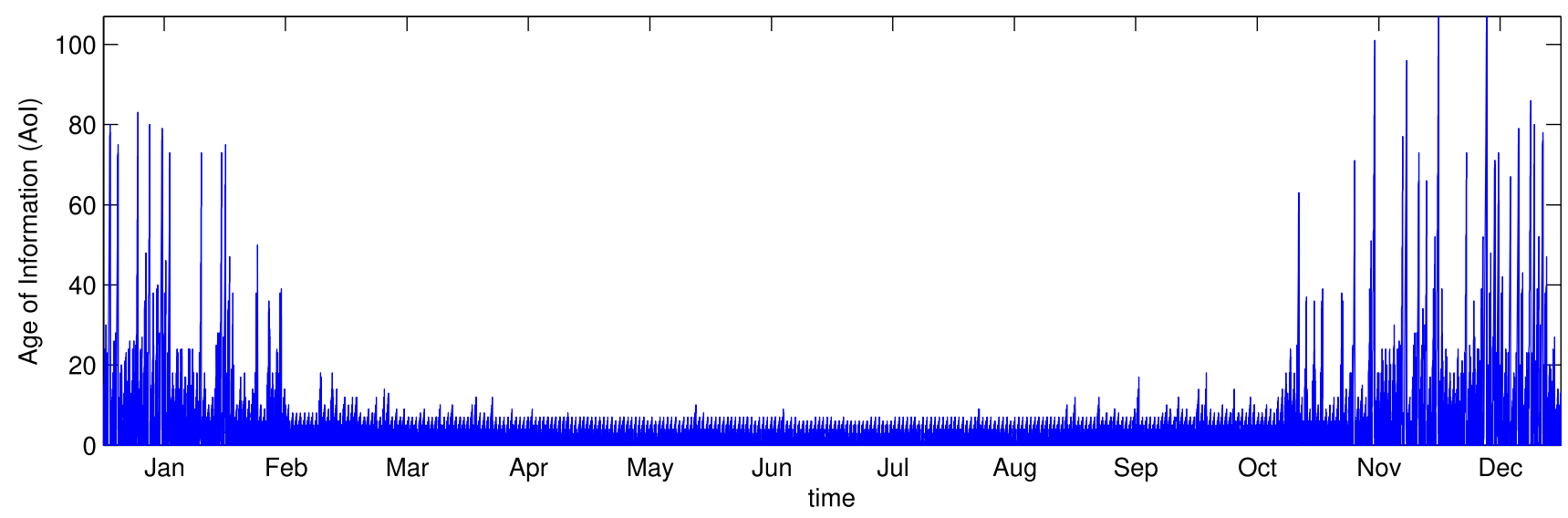

(b) MSEM-4

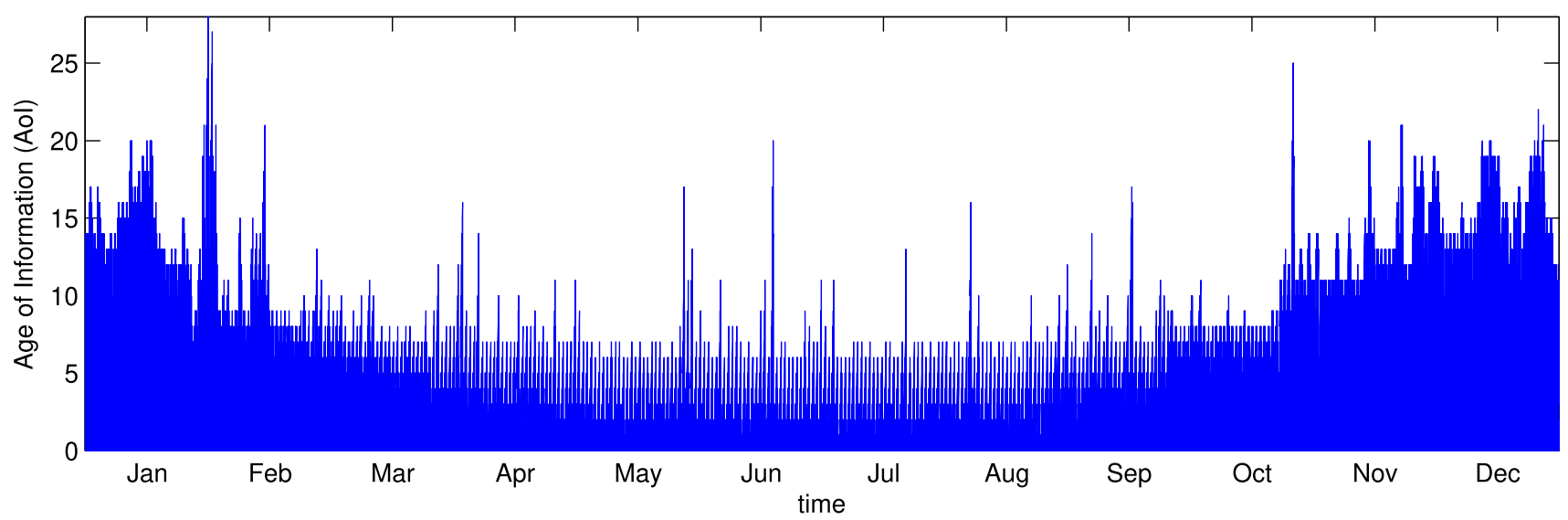

Fig. 7 The AoI process $\Delta_{k}$ depicted as a function of time for the dataset SEA for the particular case $L_{s}=5, B=100$, and $N=4$ obtained by simulations according to the solar radiation data of the year 2010 a SEM-4 b MSEM-4

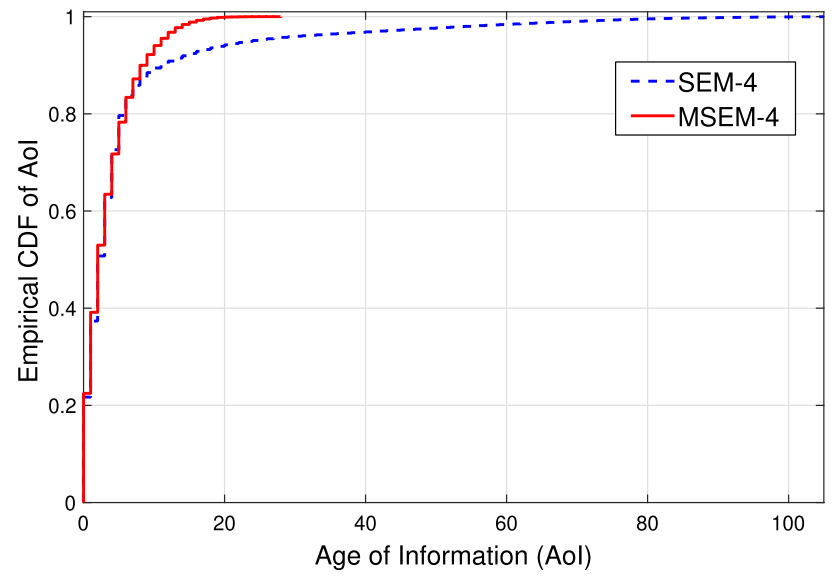

Fig. 8 Empirical CDF of AoI for the dataset SEA

whereas the month of year information is not used at all for SEM- $N$. The second main observation is related to the performance of both policies SEM- $N$ and MSEM- $N$ (in terms of AAoI reduction) increasing with increasing partitioning parameter $N$. However, in most of the scenarios, performance improvement beyond $N=4$ is minuscule for MSEM- $N$ with the exception of the $L_{s}=20$ case when further partitioning presents slight reduction in AAoI. However, note that choice of larger partitioning parameter $N$ gives rise to longer off-line computation times for the underlying MDP but more importantly, to larger storage requirements for the IoT device. As a consequence of this trade-off, for the majority of the remaining numerical examples, the partitioning parameter $N$ will be fixed to 4.

When $N=4$, the percentage reduction in AAoI obtained with MSEM-4 with respect to SEM-4 is tabulated in Table 1 for two values of $B$ and four values of $L_{s}$. We conclude the following:

- For fixed $B$, the relative improvement with MSEM-4 in AAoI increases with reduced $L_{s}$. 
Table 2 The yearly AoI violation probabilities

$\operatorname{Pr}\left\{\Delta_{k}>d\right\}$ for two values of $d=20,50$ obtained with four energy managers SEM-1, SEM4, MSEM-1, and MSEM-4 for a span of twenty years

\begin{tabular}{|c|c|c|c|c|c|c|c|c|}
\hline \multirow[t]{2}{*}{ Year } & \multicolumn{4}{|c|}{$\operatorname{Pr}\left\{\Delta_{k}>20\right\}$} & \multicolumn{4}{|c|}{$\operatorname{Pr}\left\{\Delta_{k}>50\right\}$} \\
\hline & SEM-1 & SEM-4 & MSEM-1 & MSEM-4 & SEM-1 & SEM-4 & MSEM-1 & MSEM-4 \\
\hline 1991 & .2481 & .0649 & .1458 & .0037 & .1293 & .0185 & .0386 & 0 \\
\hline 1992 & .2401 & .0554 & .1267 & .0015 & .1265 & .0150 & .0300 & 0 \\
\hline 1993 & .2230 & .0358 & .1042 & .0004 & .1060 & .0096 & .0172 & 0 \\
\hline 1994 & .2339 & .0577 & .1218 & .0028 & .1227 & .0176 & .0254 & 0 \\
\hline 1995 & .2323 & .0448 & .1117 & .0007 & .1123 & .0136 & .0224 & 0 \\
\hline 1996 & .2518 & .0719 & .1443 & .0029 & .1313 & .0217 & .0351 & 0 \\
\hline 1997 & .2596 & .0522 & .1449 & .0024 & .1315 & .0156 & .0324 & 0 \\
\hline 1998 & .2641 & .0596 & .1489 & .0010 & .1318 & .0178 & .0325 & 0 \\
\hline 1999 & .2491 & .0592 & .1351 & .0012 & .1286 & .0171 & .0305 & 0 \\
\hline 2000 & .2308 & .0339 & .1039 & .0002 & .1100 & .0103 & .0186 & 0 \\
\hline 2001 & .2376 & .0437 & .1138 & .0002 & .1171 & .0118 & .0227 & 0 \\
\hline 2002 & .2449 & .0557 & .1303 & .0004 & .1243 & .0127 & .0294 & 0 \\
\hline 2003 & .2390 & .0479 & .1147 & .0001 & .1217 & .0109 & .0240 & 0 \\
\hline 2004 & .2473 & .0606 & .1283 & .0004 & .1257 & .0170 & .0242 & 0 \\
\hline 2005 & .2355 & .0407 & .1122 & .0001 & .1111 & .0103 & .0196 & 0 \\
\hline 2006 & .2463 & .0586 & .1296 & .0013 & .1243 & .0165 & .0260 & 0 \\
\hline 2007 & .2531 & .0474 & .1256 & .0001 & .1264 & .0114 & .0221 & 0 \\
\hline 2008 & .2417 & .0475 & .1190 & .0008 & .1190 & .0128 & .0220 & 0 \\
\hline 2009 & .2384 & .0495 & .1187 & .0015 & .1194 & .0148 & .0242 & 0 \\
\hline 2010 & .2529 & .0518 & .1309 & .0006 & .1248 & .0145 & .0272 & 0 \\
\hline Avg. & .2435 & .0520 & .1256 & .0012 & .1222 & .0145 & .0263 & 0 \\
\hline
\end{tabular}

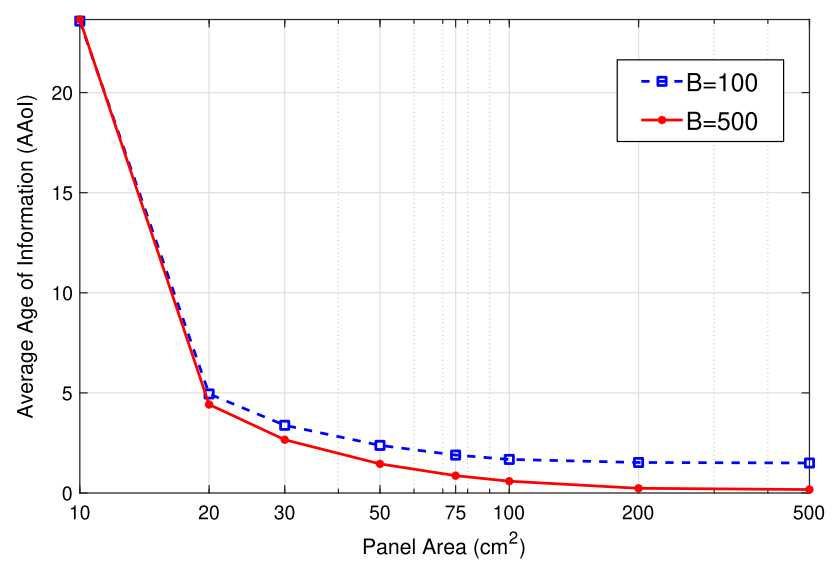

Fig. 9 The AAoI depicted as a function of the solar panel area for the dataset SEA when $L_{s}=5$ and $N=4$, for two different values of $B$

- For fixed $L_{s}$, the relative improvement with MSEM-4 in AAoI is only slight for increased $B$ except for the case $L_{s}=1$.

- The AAoI reduction with the policy MSEM-4 relative to SEM-4 is more apparent for the dataset SEA which offers more seasonal variations due to the higher latitude of the corresponding location.

We also present a snapshot of the instantaneous AoI, i.e., $\Delta_{k}, k \geq 0$ in Fig. 7 which is depicted as a function of the time index $k$, for the dataset SEA and for the particular scenario $L_{s}=5, B=100$, and $N=4$ obtained by simulations according to the solar radiation data of the year 2010 . Figure 7 reveals that monthly-switched SEM not only reduces the average AAoI but also substantially reduces the peak AoI values that appear to exist on Winter days when the solar radiation is lower. Note that a peak AoI value of 100 (exceeded several times with SEM-4) amounts to slightly more than two thirds of a day which may be quite harmful for the underlying status update application. On the contrary, for the same year, the AoI value exceeded 25 only for one single instance throughout the year which shows that MSEM-4 also significantly improves upon the peak AoI values as well. To further elaborate on the tail distribution of the AoI, we provide the empirical CDF of the AoI for the dataset SEA in Fig. 8 obtained through simulations over twenty years when SEM-4 and MSEM-4 are used as the energy management policies for the same example. We observe that with MSEM-4, the tail distribution of the AoI is improved significantly when compared to SEM-4. Although minimizing the AAoI has received considerable attention in the literature, some applications impose stricter requirements on the tail of the AoI distribution [13]. For a different view, we provide the yearly (per annum) AoI violation probability $\operatorname{Pr}\left\{\Delta_{k}>d\right\}$ for two values of the violation threshold $d=20,50$ obtained with 
four energy managers SEM-1, SEM-4, MSEM-1, and MSEM-4 for a span of twenty years in Table 2 from 1991 to 2010. Using four time intervals in a given day in the Markov model and producing an energy management policy for each of the 12 months of the year as in MSEM-4 appears to be very effective in keeping the AoI violation probability low and also throughout each of the 20 years constituting the entire simulation period. With MSEM-4, the AoI did not exceed 50 (corresponding to $500 \mathrm{~min}=8.33 \mathrm{~h}$ ) even once in a 20 year simulation run. Again when MSEM-4 is employed, the AoI exceeded 20 (corresponding to $200 \mathrm{mins}=3.33 \mathrm{~h}$ ) only in $0.12 \%$ of the cases which is a remarkable improvement over the other variants of the same algorithm which either do not use the month of year information (SEM-1 and SEM-4) or the time of day information (SEM-1 and MSEM-1). Moreover, such behavior is shown to be even across the years in the 20-years long time window demonstrating robustness of the proposed MSEM-4 energy management policy (Table 2).

As a final example, we plot in Fig. 9 the AAoI as a function of the solar panel area when MSEM-4 is deployed on the status update system with $L_{s}=5$ for two different values of $B$ for the dataset SEA. For a fixed battery capacity, the AAoI decreases as a function of increased panel area but beyond a certain value of the panel area, the reduction in AAoI is non-significant. This example shows that a suitable combination of the panel area as well as the battery capacity need to be used to attain a desirable AAoI.

\section{Conclusions}

In this paper, we study the optimal transmission policies for solar-powered IoT devices with the goal of minimizing the Average Age of Information (AAoI). Using historical datasets, we employ DTMC models for intra-day variations of harvestable solar energy and subsequently use the average reward MDP framework to obtain harvestingaware energy management policies. When making a transmission decision, these proposed policies take into account of the instantaneous AoI, the instantaneous battery level, as well as the time of day and the month of year. We show that the obtained policies are effective in AAoI reduction and also in improving upon the tail distribution of AoI. A time of day resolution of 4 partitions per day is deemed to provide satisfactory results for a wide range of scenarios. Depending on the latitude of the location for the corresponding dataset, using the month of year information in energy management policies results in up to about $85 \%$ reduction in AAoI. Future work will consist of the study of non-solar powered energy harvesting sources as well as other model-free optimization techniques such as reinforcement learning for AAoI reduction.

\section{References}

1. Abd-Elmagid, M. A., \& Dhillon, H. S. (2019). Average peak ageof-information minimization in UAV-assisted IoT networks. IEEE Transactions on Vehicular Technology, 68(2), 2003-2008.

2. Adu-Manu, K. S., Adam, N., Tapparello, C., Ayatollahi, H., \& Heinzelman, W. (2018). Energy-harvesting wireless sensor networks (EH-WSNs): A review. ACM Transactions on Sensor Networks, 14(2), 10:1-10:50.

3. Akyildiz, I., Su, W., Sankarasubramaniam, Y., \& Cayirci, E. (2002). A survey on sensor networks. IEEE Communications Magazine, 40(8), 102-114.

4. Alsheikh, M. A., Hoang, D. T., Niyato, D., Tan, H., \& Lin, S. (2015). Markov decision processes with applications in wireless sensor networks: A survey. IEEE Communications Surveys Tutorials, 17(3), 1239-1267.

5. Arafa, A., \& Ulukus, S. (2017). Age minimization in energy harvesting communications: Energy-controlled delays. In 2017 51 st Asilomar conference on signals, systems, and computers (pp. 1801-1805).

6. Arafa, A., Yang, J., \& Ulukus, S. (2018) Age-minimal online policies for energy harvesting sensors with random battery recharges. In 2018 IEEE international conference on communications (ICC) (pp. 1-6).

7. Bacinoglu, B. T., \& Uysal-Biyikoglu, E. (2017). Scheduling status updates to minimize age of information with an energy harvesting sensor. In 2017 IEEE international symposium on information theory (ISIT) (pp. 1122-1126).

8. Bengheni, A., Didi, F., \& Bambrik, A. (2019). EEM-EHWSN: enhanced energy management scheme in energy harvesting wireless sensor networks. Wireless Networks, 25, 3029-3046.

9. Buratti, C., Conti, A., Dardari, D., \& Verdone, R. (2009). An overview on wireless sensor networks technology and evolution. Sensors, 9(9), 6869-6896.

10. Castagnetti, A., Pegatoquet, A., Belleudy, C., \& Auguin, M. (2012). A framework for modeling and simulating energy harvesting WSN nodes with efficient power management policies. EURASIP Journal on Embedded Systems, 1, 8.

11. Centenaro, M., Vangelista, L., Zanella, A., \& Zorzi, M. (2016). Long-range communications in unlicensed bands: The rising stars in the IoT and smart city scenarios. IEEE Wireless Communications, 23(5), 60-67.

12. Cha, M., Kim, M., Kim, M., \& Choo, H. (2011). Adaptive dutycycling based on group size for energy balance of sensor nodes in wireless sensor networks. In Proceedings of the 2011 ACM symposium on research in applied computation, ACM, New York, NY, USA, RACS'11 (pp. 135-140).

13. Champati, J. P., Al-Zubaidy, H., \& Gross, J. (2018). Statistical guarantee optimization for age of information for the D/G/1 queue. In IEEE INFOCOM 2018-IEEE conference on computer communications workshops (INFOCOM WKSHPS) (pp. 130-135).

14. Deng, F., Yue, X., Fan, X., Guan, S., Xu, Y., \& Chen, J. (2019). Multisource energy harvesting system for a wireless sensor network node in the field environment. IEEE Internet of Things Journal, 6(1), 918-927.

15. Devillers, B., \& Gndz, D. (2012). A general framework for the optimization of energy harvesting communication systems with 
battery imperfections. Journal of Communications and Networks, 14(2), 130-139.

16. Feng, S., \& Yang, J. (2018). Optimal status updating for an energy harvesting sensor with a noisy channel. In IEEE INFOCOM 2018-IEEE conference on computer communications workshops (INFOCOM WKSHPS) (pp. 348-353).

17. Gelenbe, E., \& Zhang, Y. (2019). Performance optimization with energy packets. IEEE Systems Journal, 13(4), 3770-3780.

18. Gorlatova, M., Wallwater, A., \& Zussman, G. (2013). Networking low-power energy harvesting devices: Measurements and algorithms. IEEE Transactions on Mobile Computing, 12(9), 1853-1865.

19. Gosavi, A. (2015). Simulation-based optimization parametric optimization techniques and reinforcement learning (2nd ed.). Springer.

20. Gu, Y., Zhu, T., \& He, T. (2009) ESC: Energy synchronized communication in sustainable sensor networks. In 2009 17th IEEE international conference on network protocols (pp. 52-62).

21. Harrison, P. G., \& Patel, N. M. (2018). Optimizing energy-performance trade-offs in solar-powered edge devices. In Proceedings of the 2018 ACM/SPEC international conference on performance engineering, ACM, New York, NY, USA, ICPE'18 (pp. 253-260).

22. Heinzelman, W. B., Murphy, A. L., Carvalho, H. S., \& Perillo, M. A. (2004). Middleware to support sensor network applications. IEEE Network, 18(1), 6-14.

23. Howard, R. A. (1960). Dynamic programming and Markov processes. MIT Press.

24. Hsu, J., Zahedi, S., Kansal, A., Srivastava, M., \& Raghunathan, V. (2006) Adaptive duty cycling for energy harvesting systems. In Proceedings of the 2006 international symposium on low power electronics and design, ACM, New York, NY, USA, ISLPED'06 (pp. 180-185).

25. Huang, L., \& Modiano, E. (2015). Optimizing age-of-information in a multi-class queueing system. In 2015 IEEE international symposium on information theory (ISIT) (pp. 1681-1685).

26. Ingenu. (2016). How RPMA works: The making of RPMA. Ebook by Ingenu.

27. Jawad, H., Nordin, R., Gharghan, S., Jawad, A., \& Ismail, M. (2017). Energy-efficient wireless sensor networks for precision agriculture: A review. Sensors, 17(8), 66.

28. Kansal, A., Hsu, J., Zahedi, S., \& Srivastava, M. B. (2007). Power management in energy harvesting sensor networks. ACM Transactions on Embedded Computing Systems, 6(4), 27.

29. Kaul, S., Gruteser, M., Rai, V., \& Kenney, J. (2011). Minimizing age of information in vehicular networks. In 2011 8th Annual IEEE communications society conference on sensor, mesh and ad hoc communications and networks (pp. 350-358).

30. Kaul, S., Yates, R., \& Gruteser, M. (2012). Real-time status: How often should one update? In 2012 Proceedings IEEE INFOCOM (pp. 2731-2735).

31. Kaul, S. K., Yates, R. D., \& Gruteser, M. (2012). Status updates through queues. In 2012 46th Annual conference on information sciences and systems (CISS) (pp. 1-6).

32. Kaur, P., Singh, P., \& Sohi, B. S. (2019). Adaptive MAC protocol for solar energy harvesting based wireless sensor networks in agriculture. Wireless Personal Communications. https://doi.org/ 10.1007/s11277-019-06985-9.

33. Khan, J. A., Qureshi, H. K., \& Iqbal, A. (2015). Energy management in wireless sensor networks: A survey. Computers and Electrical Engineering, 41, 159-176.

34. Kosta, A., Pappas, N., \& Angelakis, V. (2017). Age of information: A new concept, metric, and tool. Foundations and Trends in Networking, 12(3), 162-259.

35. Ku, M., Chen, Y., \& Liu, K. J. R. (2015). Data-driven stochastic models and policies for energy harvesting sensor communications. IEEE Journal on Selected Areas in Communications, 33(8), 1505-1520.

36. Liu, H., Chandra, A., \& Srivastava, J. (2006) eSENSE: Energy efficient stochastic sensing framework scheme for wireless sensor platforms. In Proceedings of the 5th international conference on information processing in sensor networks, ACM, New York, NY, USA, IPSN'06 (pp. 235-242).

37. Margelis, G., Piechocki, R., Kaleshi, D., \& Thomas, P. (2015). Low throughput networks for the IoT: Lessons learned from industrial implementations. In 2015 IEEE 2nd world forum on internet of things (WF-IoT) (pp. 181-186).

38. Michelusi, N., Stamatiou, K., \& Zorzi, M. (2013). Transmission policies for energy harvesting sensors with time-correlated energy supply. IEEE Transactions on Communications, 61(7), 2988-3001.

39. Mikhaylov, K., Petaejaejaervi, J., \& Haenninen, T. (2016). Analysis of capacity and scalability of the LoRa low power wide area network technology. In 22th European wireless conference on European wireless 2016 (pp. 119-124).

40. Moser, C., Chen, J. J., \& Thiele, L. (2008). An energy management framework for energy harvesting embedded systems. Journal on Emerging Technologies in Computing Systems, 6(2), $7: 1-7: 21$.

41. National Renewable Energy Laboratory. (2018). National solar radiation database. Retrieved August 15, 2018, from https:// rredc.nrel.gov/solar/old_data/nsrdb.

42. Nguyen, D. T., \& Le, L. B. (2014). Optimal bidding strategy for microgrids considering renewable energy and building thermal dynamics. IEEE Transactions on Smart Grid, 5(4), 1608-1620.

43. Pappas, N., Gunnarsson, J., Kratz, L., Kountouris, M., \& Angelakis, V. (2015) Age of information of multiple sources with queue management. In 2015 IEEE international conference on communications (ICC) (pp. 5935-5940).

44. Reddy, S., \& Murthy, C. R. (2010). Profile-based load scheduling in wireless energy harvesting sensors for data rate maximization. In 2010 IEEE international conference on communications (pp. $1-5)$.

45. Sharma, A., \& Kakkar, A. (2019). Machine learning based optimal renewable energy allocation in sustained wireless sensor networks. Wireless Networks, 25, 3953-3981.

46. Sharma, V., Mukherji, U., Joseph, V., \& Gupta, S. (2010). Optimal energy management policies for energy harvesting sensor nodes. IEEE Transactions on Wireless Communications, 9(4), 1326-1336.

47. Sinha, A., \& Chandrakasan, A. (2001). Dynamic power management in wireless sensor networks. IEEE Design Test of Computers, 18(2), 62-74.

48. Stamatakis, G., Pappas, N., \& Traganitis, A. (2018). Optimal policies for status update generation in a wireless system with heterogeneous traffic. CoRR abs/1810.03201, arxiv:1810.03201.

49. Sun, Y., Uysal-Biyikoglu, E., Yates, R. D., Koksal, C. E., \& Shroff, N. B. (2017). Update or wait: How to keep your data fresh. IEEE Transactions on Information Theory, 63(11), $7492-7508$

50. Sutton, R. S., \& Barto, A. G. (2018). Introduction to reinforcement learning (2nd ed.). MIT Press.

51. Swapna Kumar, S., \& Kashwan, K. (2013). Research study of energy harvesting in wireless sensor networks. International Journal of Renewable Energy Research, 3, 745-753.

52. Tripathi, V., Talak, R., \& Modiano, E. (2019). Age of information for discrete time queues. CoRR abs/1901.10463, arxiv:1901. 10463.

53. Tunc, C., \& Akar, N. (2017). Markov fluid queue model of an energy harvesting IoT device with adaptive sensing. Performance Evaluation, 111, 1-16. 
54. Tutuncuoglu, K., \& Yener, A. (2012). Optimum transmission policies for battery limited energy harvesting nodes. IEEE Transactions on Wireless Communications, 11(3), 1180-1189.

55. Tutuncuoglu, K., Yener, A., \& Ulukus, S. (2015). Optimum policies for an energy harvesting transmitter under energy storage losses. IEEE Journal on Selected Areas in Communications, 33(3), 467-481.

56. Ulukus, S., Yener, A., Erkip, E., Simeone, O., Zorzi, M., Grover, P., \& Huang, K. (2015). Energy harvesting wireless communications: A review of recent advances. IEEE Journal on Selected Areas in Communications, 33(3), 360-381.

57. Vigorito, C. M., Ganesan, D., Barto, A. G. (2007). Adaptive control of duty cycling in energy-harvesting wireless sensor networks. In 2007 4th Annual IEEE communications society conference on sensor, mesh and ad hoc communications and networks (pp. 21-30).

58. Wu, X., Yang, J., \& Wu, J. (2018). Optimal status update for age of information minimization with an energy harvesting source. IEEE Transactions on Green Communications and Networking, 2(1), 193-204. https://doi.org/10.1109/TGCN.2017.2778501.

59. Xu, G., Shen, W., \& Wang, X. (2014). Applications of wireless sensor networks in marine environment monitoring: A survey. Sensors, 14(9), 16932-16954.

60. Yang, J., \& Ulukus, S. (2012). Optimal packet scheduling in an energy harvesting communication system. IEEE Transactions on Communications, 60(1), 220-230.

61. Zhang, S. (2013). Modeling, analysis and design of energy harvesting communication systems. $\mathrm{PhD}$ thesis, Dept. of Electrical and Computer Engineering, University of Rochester.

62. Zhou, G., Huang, L., Li, W., \& Zhu, Z. (2014). Harvesting ambient environmental energy for wireless sensor networks: A survey. Journal of Sensors, 2014, 1-20.

63. Zhu, T., Zhong, Z., Gu, Y., He, T., \& Zhang, Z. L. (2009) Leakage-aware energy synchronization for wireless sensor networks. In Proceedings of the 7th international conference on mobile systems, applications, and services, ACM, New York, NY, USA, MobiSys '09 (pp. 319-332).

Publisher's Note Springer Nature remains neutral with regard to jurisdictional claims in published maps and institutional affiliations.

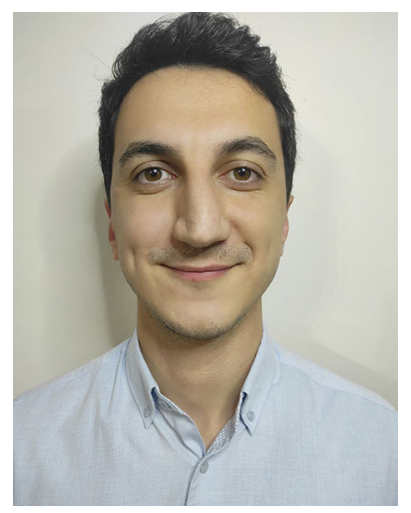

Abdul Kerim Aydin received his B.S. and M.S. degrees from Bilkent University, Ankara, Turkey, in 2015 and 2018, respectively, both in electrical and electronics engineering. $\mathrm{He}$ is currently an embedded software engineering specialist at ASELSAN, Ankara, Turkey and his research interests include wireless networks, embedded systems, and particularly onboard railway signalling and train control and management systems.

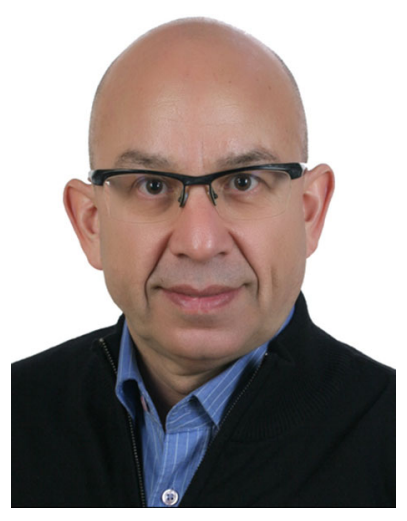

Nail Akar received his B.S. degree from Middle East Technical University, Turkey, in 1987 and M.S. and Ph.D. degrees from Bilkent University, Ankara, Turkey, in 1989 and 1994, respectively, all in electrical and electronics engineering. From 1994 to 1996, he was a visiting scholar and a visiting assistant professor in the Computer Science Telecommunications program at the University of MissouriKansas City, USA. He joined the Technology Planning and Integration group at Long Distance Division, Sprint, Overland Park, Kansas, in 1996, where he held a senior member of technical staff position from 1999 to 2000. Since 2000, he has been with Bilkent University currently as a Professor of the Electrical and Electronics Engineering Department and as the Associate Dean of the Engineering Faculty. He visited the School of Computing, University of Missouri-Kansas City, as a Fulbright scholar in 2010 for a period of six months. His research interests are on performance modeling of computer and communication systems and networks, wireless networks, Internet of Things, queueing theory, and optimization.. 